\title{
Minimal Residual Disease after Conventional Treatment Significantly Impacts on Progression-Free Survival of Patients with Follicular Lymphoma: The FIL FOLL05 Trial
}

\author{
Sara Galimberti ${ }^{1}$, Stefano Luminari ${ }^{2}$, Elena Ciabatti ${ }^{1,3}$, Susanna Grassi ${ }^{1}$, Francesca Guerrini ${ }^{1}$, \\ Alessandra Dondi ${ }^{2}$, Luigi Marcheselli ${ }^{2}$, Marco Ladetto ${ }^{4}$, Pier Paolo Piccaluga ${ }^{5}$, Anna Gazzola ${ }^{5}$, \\ Claudia Mannu', Luigia Monitillo ${ }^{4}$, Barbara Mantoan ${ }^{4}$, Ilaria Del Giudice ${ }^{6}$, Irene Della Starza ${ }^{6}$, \\ Marzia Cavalli ${ }^{6}$, Luca Arcaini ${ }^{7}$, Alessandra Tucci ${ }^{8}$, Giuseppe Alberto Palumbo ${ }^{9}$, Luigi Rigacci ${ }^{10}$, \\ Alessandro Pulsoni ${ }^{6}$, Umberto Vitolo ${ }^{4}$, Carola Boccomini ${ }^{4}$, Daniele Vallisa ${ }^{11}$, Giovanni Bertoldero ${ }^{12}$, \\ Gianluca Gaidano ${ }^{13}$, Pellegrino Musto ${ }^{14}$, Mario Petrini ${ }^{1}$, and Massimo Federico ${ }^{2}$
}

\section{Abstract}

Purpose: The role of the minimal residual disease (MRD) in follicular lymphoma is still debated. In this study, we assessed whether the BCL2/IGH rearrangement could have a prognostic role in patients receiving R-CHOP, R-FM, or R-CVP.

Experimental Design: DNAs from 415 patients among the 504 cases enrolled in the FOLL05 trial (NCT00774826) were centralized and assessed for the BCL2/IGH at diagnosis, at the end of treatment, and after 12 and 24 months.

Results: At diagnosis, the molecular marker was detected in 53\% of cases. Patients without molecular marker or with a low molecular tumor burden $\left(<1 \times 10^{-4}\right.$ copies $)$ showed higher complete remission (CR) rate and longer progression-free survival (PFS; 3-year PFS $80 \%$ vs. 59\%; $P=0.015$ ). PFS was significantly conditioned by the PCR status at 12 and 24 months, with 3 -year PFS of $66 \%$ for MRD ${ }^{-}$cases versus $41 \%$ for those $\mathrm{MRD}^{+}$at 12 months $(P=0.015)$, and $84 \%$ versus $50 \%$ at 24 months $(P=0.014)$. The MRD negativity at 12 and 24 months resulted in an improved PFS both in CR and in partial remission (PR) patients (3-year $\mathrm{PFS}=72 \%$ for cases $\mathrm{CR} / \mathrm{PCR}^{-}$vs. $32 \%$ for those $\mathrm{CR} / \mathrm{PCR}^{+}$vs. $62 \%$ for those $\mathrm{PR} / \mathrm{PCR}^{-}$and $25 \%$ for patients in $\left.\mathrm{PR} / \mathrm{PCR}^{+} ; \mathrm{P}=0.001\right)$. The prognostic value of $\mathrm{MRD}$ at 12 and 24 months of follow-up was confirmed also in multivariate analysis.

Conclusions: In this study, standardized molecular techniques have been adopted and applied on bone marrow samples from a large cohort. Data reported show that the MRD detection is a powerful independent predictor of PFS in patients with follicular lymphoma receiving conventional chemoimmunotherapy. Clin Cancer Res; 20(24); 6398-405. (C2014 AACR.

\section{Introduction}

The monitoring of minimal residual disease (MRD) in follicular lymphoma is a well-established predictor of outcome in the autologous transplantation scenario, in which the negative impact on survival of patients

${ }^{1}$ Department of Clinical and Experimental Medicine - University of Pisa, Pisa, Italy. ${ }^{2}$ Department of Diagnostic, Clinics and Public Health, University of Modena and Reggio Emilia, Modena, Italy. ${ }^{3}$ GenOMec School of University of Siena, Italy. ${ }^{4}$ Hospital of Science and Health City, Turin, Italy. ${ }^{5}$ Department of Experimental, Diagnostic, and Speciality Medicine, Bologna University, Bologna, Italy. ${ }^{6}$ Hematology, Department of Cellular Biotechnologies and Hematology, Sapienza University, Rome, Italy. ${ }^{7}$ Department of Oncology and Hematology, IRCSS "San Matteo", University of Pavia, Italy. ${ }^{8}$ Hematology Unit, Hospital of Brescia, Brescia, Italy. ${ }^{9} \mathrm{Hema}-$ tology Unit, "Ferrarotto" Hospital, Catania, Italy. ${ }^{10} \mathrm{Hematology}$ Unit, AO Careggi, Florence, Italy. ${ }^{11} \mathrm{Hematology}$ Unit, Piacenza Hospital, Piacenza, Italy. ${ }^{12}$ Hematology Unit, Merano Hospital, Merano, Italy. ${ }^{13}$ SCDU Hematology, Department of Translational Medicine, University of East Piedmont, Novara, Italy. ${ }^{14}$ Scientific Direction, IECCS, Referral Cancer Center of basilicata, Rionero In vulture (Pz), Italy. receiving $\mathrm{BCL} 2 / \mathrm{IGH}^{+}$autologous stem cells and of the MRD persistence after transplantation have been already demonstrated (1-3). On the contrary, the role of MRD after conventional treatments is still debated $(4,5)$. The first critical point is what is the best technique for MRD assessment; indeed, more than half of patients affected by

Note: Supplementary data for this article are available at Clinical Cancer Research Online (http://clincancerres.aacrjournals.org/).

Prior presentation: The results of this study have been presented at the 2012 American Society of Hematology and at 2012 EHA annual meetings.

Corresponding Author: Sara Galimberti, Division of Hematology, Department of Clinical and Experimental Medicine, University of Pisa, Via Roma, 67, Pisa, PI 56126, Italy. Phone: +39-050-993486; Fax: 39-050-993378; E-mail: sara.galimberti@med.unipi.it

doi: 10.1158/1078-0432.CCR-14-0407

(c)2014 American Association for Cancer Research. 


\section{Translational Relevance}

Follicular lymphoma represents an indolent lymphoproliferative disease, but the incidence of relapse still interests more than one third of the responsive patients. Thus, the introduction of the rituximab maintenance and the monitoring of the minimal residual disease (MRD) could be useful for starting a preemptive therapy and delaying the clinical relapse. In this study, we assessed 415 patients affected by follicular lymphoma receiving R-CHOP, R-FM, or R-CVP by qualitative and quantitative PCR for BCL2/IGH rearrangement. We showed that: (i) the presence of the BCL2/IGH rearrangement in the bone marrow (BM) at diagnosis has got a predictive value on progression-free survival (PFS); (ii) a low molecular tumor burden at diagnosis positively impacts on the quality of response and PFS; (iii) the MRD negativity after 12 and 24 months off treatment correlates with a better outcome; (iv) R-CVP is the regimen offering a lower molecular disease clearance in comparison with R-CHOP and R-FM. Thus, a molecular assessment during the work-up of patients with follicular lymphoma could be considered as a sort of "dynamic" risk score that could lead to treat by rituximab patients losing the MRD negativity or to avoid maintenance in patients at very low risk of relapse.

follicular lymphoma carry the $\mathrm{t}(14 ; 18)(\mathrm{q} 32 ; \mathrm{q} 21)$ and the correspondent fusion gene BCL2/IGH that could be easily detectable by the PCR (6). Usually, the breakpoint is inside the major breakpoint region (MBR; ref. 7), whereas most of the remaining cases show the rupture in other regions, namely the minor cluster region $(\mathrm{mcr})$, and the $3^{\prime} \mathrm{MBR}$ (8). Breakpoints occurring in the MBR can be used for MRD purposes using standardized qualitative and quantitative PCR reactions (9), whereas PCR-based assays for translocations occurring at other breakpoints have not been yet extensively validated (10). In the remaining cases, patient-specific rearrangement of the immunoglobulins heavy chains (IGH) could be also detected, but this method is expensive, time-consuming, and offers a lower sensitivity (11).

The second issue concerns the best timing for the MRD assessment. In particular, it is unclear whether MRD would play any predictive role already at the end of treatment, or if its significance would be higher during the follow-up, before or after autotransplantation, or during the rituximab maintenance.

The introduction of rituximab $(\mathrm{R})$ in the clinical practice and the possibility of consolidation with ${ }^{90} \mathrm{Y}$-ibritumomab significantly increased the probability of achieving the MRD eradication $(12,13)$.

van Oers and colleagues (14) showed that the BCL2/ IGH-positive status before treatment with R-CHOP in relapsed/resistant patients did negatively condition the progression-free survival (PFS). In another study conducted by the Italian Lymphoma Group, MRD resulted as a powerful outcome predictor in patients receiving rituximab maintenance (15).

Moreover, after the introduction of the qPCR during the last decade, the role of the "molecular tumor burden" has been also evaluated: Rambaldi and colleagues (16) reported that $70 \%$ of patients with low amount of BCL2/IGH copies achieved complete remission (CR) compared with only $26 \%$ of those with higher BCL2/IGH levels, with a significant advantage on the event-free survival.

Thus, in the 2005 the Fondazione Italiana Linfomi (FIL) decided to assess the MRD in patients with follicular lymphoma enrolled in the large phase III multicenter study FOLL05 (NCT00774826). In this trial, conducted between March 2006 and September 2010, 534 untreated patients affected by advanced follicular lymphoma were randomized to receive $\mathrm{R}-\mathrm{CHOP}$ (that resulted to be the best regimen), R-CVP, or R-FM, as previously reported (17).

Here, we present the results of the molecular assessment of patients enrolled in the FOLL05 trial providing new insights on some still open issues about MRD in patients with follicular lymphoma receiving chemoimmunotherapy.

\section{Patients and Methods}

\section{Study design and treatment}

The prospective, randomized, multicenter phase III trial FOLL05 (NCT00774826) was conducted in 58 Italian centers, in accordance to the Declaration of Helsinki. The clinical trial included previously untreated patients, aged 18 to 75 years, with a histologic confirmed diagnosis of follicular lymphoma grade 1, 2, and 3a, Ann Arbor stages II to IV, ECOG (Eastern Cooperative Oncology Group) performance status 0 to 2, and active disease (18). In addition to the physical examination and total body CT scan, before enrollment all patients underwent bone marrow (BM) biopsy and aspirate for assessment of the BCL2/IGH fusion gene. Central pathology review was performed for all grade 3 follicular lymphomas or when the local pathologist did not specify grading. In each center all BM biopsies were assessed by immunohistochemistry (at least CD20, CD10, and CD5), to confirm the morphologic diagnosis of follicular lymphoma. All patients underwent an intermediate CT scan for assessment of response after cycle 3 and at treatment completion. Clinical response assessment was performed with physical examination, laboratory tests, and total body CT scan; BM biopsy and aspirate were required only for patients with initial BM involvement or BCL2/IGH positivity. Quality of response was defined according to the standardized international criteria (19). The conversion of the MRD negativity to the MRD positivity was not considered as relapse in the computation of PFS.

\section{Molecular assays}

Qualitative BCL2/IGH rearrangement analysis was planned at baseline, at 6 weeks after the end of treatment, and then every 6 months during the second and third year of 
follow-up. All qualitative molecular analyses were centralized at the molecular laboratory of the Division of Hematology of the Pisa University (Italy). The four laboratories composing the FIL-MRD network retrospectively performed qPCR assays at diagnosis and at the end of therapy, after the inter-laboratory standardization of the used techniques.

DNA was extracted from BM mononuclear cells by the Wizard Genomic DNA Purification Kit (Promega). To amplify BCL2/IGH rearrangement, nested PCR reactions were performed as previously described (20).

The sensitivity of the qualitative PCR assays was confirmed by testing serial dilutions of DNA derived from the BCL2/IGH-positive DOHH-2 cell line, achieving a limiting dilution of $1: 10^{-5}$. A second reaction for mcr breakpoint was also performed, as reported in literature (21).

qPCR was performed using the technique previously described by Ladetto and colleagues (22). Also in this case, standard curves were constructed using DNA extracted from the DOHH-2 cells. Even in this case, the sensitivity was $1: 10^{-5}$

To confirm the specificity of BCL2/IGH rearrangement, four-paired samples (at diagnosis and after treatment) were sequenced using the ABI Prism BigDye Terminator Cycle Sequencing Kit 1.1 and the ABI Prism 3100 Genetic Analyzer (PE Applied Biosystems).

\section{Statistical analysis}

All statistical analyses were performed using the SPSS 17.0 software (SPSS Inc.) at the FIL data center in Modena (Italy). Because about half of patients with follicular lymphoma relapse by the third year after treatment, and overall survival analyses are not possible in the time frame of the trial, PFS was chosen as the best surrogate of the efficacy of treatment. PFS was measured from the date of the study entry to the last follow-up, or to one of the following events: death from any cause, disease progression, and relapse. Survival curves were calculated using the Kaplan-Meier method, and statistical comparisons between curves were made using the log-rank test. Post-hoc comparisons were obtained using the Cox proportional hazard regression method. The $\chi^{2}$ test, Fisher exact test, and Kruskal-Wallis test were used to compare variables when appropriate. For establishing the value of quantitative BCL2/IGH rearrangement on relapse, a ROC curve was performed. All statistical comparisons were two-sided. The date of the last molecular follow-up was December 30, 2012.

\section{Results}

Qualitative PCR positivity before therapy predicts the clinical response

Five hundred and thirty-four patients were enrolled into the FOLL05 trial by 58 Italian institutions; 30 patients were subsequently excluded, and the remaining 504 patients were analyzed. DNAs from 89 cases were not centralized in Pisa. Consequently, at baseline, 415 of the 504 eligible patients (82\%) were assessed for BCL2/IGH rearrangement by qualitative PCR (see Supplementary Fig. S1): In 220 cases (53\%) the molecular marker was found. The breakpoint was in the MBR in the $92 \%$ of cases and in the mcr in the remaining $8 \%$. Overall, 227 patients were scored as showing $\mathrm{BM}$ infiltration after the local microscopy observation; in 40 of them $(17.6 \%)$, we were not able to find the BCL2/IGH, probably for the presence of a rearrangement involving other rare breakpoints On the other hand, in 127 of the 188 cases without BM infiltration at the microscopy $(67.5 \%)$ the molecular marker was found, possibly due to a submicroscopic BM involvement.

Cytogenetic data about translocation between chromosome 14 and 18 were not available for this trial.

No significant differences were observed for the main clinical and prognostic features, and for treatment allocation, between cases with and without molecular assessment at enrollment, and between patients resulting $\mathrm{PCR}^{-}$or $\mathrm{PCR}^{+}$(Tables 1 and 2).

Both PCR positivity and BM infiltration at the enrollment had a significant impact on the quality of response. The percentage of cases not achieving the complete response at the end of therapy was higher for patients $\mathrm{PCR}^{+}$or $\mathrm{BM}^{+}$in respect of cases without molecular marker or BM infiltration $\left[61.9 \%\right.$ for $\mathrm{PCR}^{+}$vs. $38.1 \%$ for $\mathrm{PCR}^{-}$ patients $(P=0.027) ; 32 \%$ for $\mathrm{BM}^{+}$vs. $21.8 \%$ for $\mathrm{BM}^{-}$cases $(P=0.021)]$

The 3-year PFS was significantly advantageous for patients $\mathrm{BM}^{-} / \mathrm{PCR}^{-}$versus those $\mathrm{BM}^{+} / \mathrm{PCR}^{+}(74 \%$ vs $55 \% ; P=0.04)$.

\section{Molecular tumor burden before therapy significantly predicts both quality of response and PFS}

At the study enrollment, the molecular tumor burden was assessed by qPCR in 105 cases of the 203 already positive for MBR breakpoint; this difference between cases assessed by qualitative and quantitative PCR was due to the residual availability of DNA. No significant differences were observed for clinical features and treatment allocation between cases with or without qPCR assessment (Table 3). The quantization of molecular tumor burden showed wide interpatients variability: the median value was $3 \times 10^{-3}$ copies, ranging from $2 \times 10^{-5}$ to 6 copies. The BCL2/IGH copy number did not correlate with stage, performance status, age ( $<$ or $>65$ year), or gender, but was significantly higher in patients presenting with high FLIPI and FLIPI2 score.

When a ROC analysis-computing BCL2/IGH copies (as continuous variable) versus relapse (as dichotomic variable) was performed, a BCL2/IGH copy number $>1 \times 10^{-4}$ was the most predictive value conditioning the quality of response and the relapse rate. Indeed, among patients with high molecular tumor burden, overall response rate (ORR) was significantly lower than in cases with low molecular tumor burden $(38.9 \%$ vs. $76.6 \% ; P=0.006)$.

Moreover, only $22 \%$ of cases showing $<1 \times 10^{-4}$ copies relapsed versus $78 \%$ of patients with $>1 \times 10^{-4}$ copies $(P=$ $0.033)$. The treatment allocation was not different between 
Table 1. Comparison of patients'

characteristics between cases assessed or not

by qualitative PCR for BCL2//GH rearrangement

Patients

Qualitative PCR

\begin{tabular}{|c|c|c|c|}
\hline \multirow[b]{2}{*}{ Characteristics } & \multicolumn{3}{|c|}{ Qualitative PCR } \\
\hline & Performed & $\begin{array}{c}\text { Not } \\
\text { performed }\end{array}$ & $P$ \\
\hline Number of patients & 415 & 106 & \\
\hline Median age, y & 69 & 68 & N.S. \\
\hline \multicolumn{4}{|l|}{ Sex } \\
\hline Male & $47 \%$ & $54 \%$ & \multirow[t]{2}{*}{ N.S. } \\
\hline Female & $53 \%$ & $46 \%$ & \\
\hline \multicolumn{4}{|l|}{ Histotype } \\
\hline Grade 1 & $25 \%$ & $35 \%$ & \multirow{4}{*}{ N.S. } \\
\hline Grade 2 & $49 \%$ & $44 \%$ & \\
\hline Grade 3a & $15 \%$ & $13 \%$ & \\
\hline Unclassified & $11 \%$ & $8 \%$ & \\
\hline \multicolumn{4}{|l|}{ Ann Arbor stage } \\
\hline II & $8 \%$ & $9 \%$ & \multirow{3}{*}{ N.S. } \\
\hline III & $28 \%$ & $29 \%$ & \\
\hline IV & $64 \%$ & $62 \%$ & \\
\hline \multicolumn{4}{|c|}{ ECOG performance status } \\
\hline$>1$ & $2.7 \%$ & $2.8 \%$ & N.S. \\
\hline \multicolumn{4}{|l|}{ FLIPI } \\
\hline $0-2$ & $58 \%$ & $64 \%$ & \multirow[t]{2}{*}{ N.S. } \\
\hline $3-5$ & $42 \%$ & $36 \%$ & \\
\hline \multicolumn{4}{|l|}{ FLIPI 2} \\
\hline $0-2$ & $64 \%$ & $75 \%$ & \multirow[t]{2}{*}{ N.S. } \\
\hline $3-5$ & $36 \%$ & $25 \%$ & \\
\hline \multicolumn{4}{|l|}{$\beta 2$-microglobulin } \\
\hline$>\mathrm{UNL}$ & $46 \%$ & $44 \%$ & N.S. \\
\hline BM involvement & $57 \%$ & $54 \%$ & N.S. \\
\hline \multicolumn{4}{|l|}{ Treatment allocation } \\
\hline R-CVP & $37 \%$ & $32 \%$ & \multirow{3}{*}{ N.S. } \\
\hline R-FM & $39 \%$ & $35 \%$ & \\
\hline $\mathrm{R}-\mathrm{CHOP}$ & $32 \%$ & $33 \%$ & \\
\hline
\end{tabular}

Abbreviation: N.S., "statistically not significant."

the two cohorts; moreover, cases displaying values $<1 \times$ $10^{-4}$ showed a clear advantage also in terms of PFS (3-year PFS $80 \%$ vs. $59 \%$ for cases with higher molecular tumor burden; $P=0.015$; Fig. 1).

In the multivariate analysis, the molecular tumor burden significance was analyzed together with FLIPI, BM involvement, quality of response [CR vs. partial remission (PR) or stable disease], and arm of therapy (R-CVP vs. R-CHOP or R-FM) A high FLIPI score, missing the $\mathrm{CR}$, and a high molecular tumor burden before therapy retained their negative impact on PFS [HR, 2.51; 95\% confidence interval (CI), 2.44-4.3; $P=0.009,0.010,0.027$, respectively; see Supplementary Table S1].

In particular, when the molecular tumor burden at diagnosis was analyzed in respect of the arm of randomization, the Mantel-Heanzel analysis confirmed that the high molecular tumor burden retained its negative impact on PFS independently from the arm of randomization (HR, 4.97; test for unequal HR: $P=0.929$ ).

\section{The impact of treatment on MRD}

At the first time point of molecular observation ( 6 weeks after the end of therapy), 3 patients dropped out from the protocol and 63 samples were not sent to the referral molecular laboratory; thus, 154 of the 220 previously $\mathrm{PCR}^{+}$cases were reassessed by qualitative PCR: 109 $(70.8 \%)$ achieved the PCR negativity.

To verify the identity of the molecular marker at the end of treatment with that observed at diagnosis, 4 patients (8 paired samples) were longitudinally sequenced: all tests confirmed the specificity of the BCL2/IGH rearrangement.

The MRD status at the end of therapy did not significantly correlate with the clinical features, quality of

Table 2. Comparison of patients' characteristics between cases with or without molecular marker at diagnosis

\begin{tabular}{|c|c|c|c|}
\hline \multirow[b]{2}{*}{ Characteristics } & \multicolumn{3}{|c|}{$\begin{array}{c}\text { Patients } \\
\text { Qualitative PCR }\end{array}$} \\
\hline & Positive & Negative & $P$ \\
\hline Number of patients & 220 & 195 & \\
\hline Median age, y & 69 & 68 & N.S. \\
\hline \multicolumn{4}{|l|}{ Sex } \\
\hline Male & $52 \%$ & $55 \%$ & \multirow[t]{2}{*}{ N.S } \\
\hline Female & $48 \%$ & $45 \%$ & \\
\hline \multicolumn{4}{|l|}{ Histotype } \\
\hline Grade 1 & $39 \%$ & $31 \%$ & \multirow{4}{*}{ N.S } \\
\hline Grade 2 & $44 \%$ & $43 \%$ & \\
\hline Grade 3a & $11 \%$ & $16 \%$ & \\
\hline Unclassified & $6 \%$ & $10 \%$ & \\
\hline \multicolumn{4}{|l|}{ Ann Arbor stage } \\
\hline$\|$ & $6 \%$ & $12 \%$ & \multirow{3}{*}{ N.S. } \\
\hline III & $28 \%$ & $30 \%$ & \\
\hline IV & $66 \%$ & $58 \%$ & \\
\hline \multicolumn{4}{|c|}{ ECOG performance status } \\
\hline$>1$ & $3.0 \%$ & $2.7 \%$ & N.S. \\
\hline \multicolumn{4}{|l|}{ FLIPI } \\
\hline $0-2$ & $56 \%$ & $45 \%$ & \multirow[t]{2}{*}{ N.S } \\
\hline $3-5$ & $44 \%$ & $55 \%$ & \\
\hline \multicolumn{4}{|l|}{ FLIPI 2} \\
\hline $0-2$ & $60 \%$ & $70 \%$ & \multirow[t]{2}{*}{ N.S. } \\
\hline $3-5$ & $40 \%$ & $30 \%$ & \\
\hline \multicolumn{4}{|l|}{$\beta 2$-microglobulin } \\
\hline$>U N L$ & $46 \%$ & $54 \%$ & N.S. \\
\hline BM involvement & $45 \%$ & $55 \%$ & N.S. \\
\hline \multicolumn{4}{|l|}{ Treatment allocation } \\
\hline R-CVP & $33 \%$ & $33 \%$ & \multirow{3}{*}{ N.S. } \\
\hline R-FM & $35 \%$ & $34 \%$ & \\
\hline $\mathrm{R}-\mathrm{CHOP}$ & $32 \%$ & $33 \%$ & \\
\hline
\end{tabular}

Abbreviation: N.S., "statistically not significant." 


\begin{tabular}{|c|c|c|c|}
\hline \multirow[b]{2}{*}{ Characteristics } & \multicolumn{3}{|c|}{$\begin{array}{l}\text { Patients } \\
\text { qPCR }\end{array}$} \\
\hline & Assessed & $\begin{array}{c}\text { Not } \\
\text { assessed }\end{array}$ & $P$ \\
\hline Number of patients & 105 & 115 & \\
\hline Median age, y & 68 & 67 & N.S. \\
\hline \multicolumn{4}{|l|}{ Sex } \\
\hline Male & $48 \%$ & $52 \%$ & N.S. \\
\hline Female & $46 \%$ & $44 \%$ & \\
\hline \multicolumn{4}{|l|}{ Histotype } \\
\hline Grade 1 & $53 \%$ & $47 \%$ & \\
\hline Grade 2 & $44 \%$ & $56 \%$ & N.S. \\
\hline Grade 3a & $46 \%$ & $54 \%$ & \\
\hline Unclassified & $33 \%$ & $67 \%$ & \\
\hline \multicolumn{4}{|l|}{ Ann Arbor stage } \\
\hline II & $50 \%$ & $50 \%$ & \\
\hline III & $51 \%$ & $49 \%$ & N.S. \\
\hline IV & $45 \%$ & $55 \%$ & \\
\hline \multicolumn{4}{|c|}{ ECOG performance status } \\
\hline$>1$ & $3.8 \%$ & $1.7 \%$ & N.S. \\
\hline \multicolumn{4}{|l|}{ FLIPI } \\
\hline $0-2$ & $54 \%$ & $46 \%$ & N.S. \\
\hline $3-5$ & $58 \%$ & $42 \%$ & \\
\hline \multicolumn{4}{|l|}{ FLIPI 2} \\
\hline $0-2$ & $56 \%$ & $44 \%$ & N.S. \\
\hline $3-5$ & $58 \%$ & $42 \%$ & \\
\hline \multicolumn{4}{|l|}{$\beta 2$-microglobulin } \\
\hline$>\mathrm{UNL}$ & $58 \%$ & $42 \%$ & N.S. \\
\hline BM involvement & $56 \%$ & $44 \%$ & N.S. \\
\hline \multicolumn{4}{|l|}{ Treatment allocation } \\
\hline R-CVP & $43 \%$ & $57 \%$ & \\
\hline R-FM & $48 \%$ & $52 \%$ & N.S. \\
\hline $\mathrm{R}-\mathrm{CHOP}$ & $50 \%$ & $50 \%$ & \\
\hline
\end{tabular}

N.S. means "statistically not significant"

response, or therapeutic arm: the percentage of cases initially $\mathrm{PCR}^{+}$that became $\mathrm{MRD}^{-}$after treatment was superimposable for patients receiving R-CHOP and R-FM (39\% and $36 \%$, respectively). Interestingly, only $25 \%$ of patients receiving R-CVP achieved the PCR negativity; even if statistically not significant $(P=0.26)$, this is in accordance to that already observed in the clinical trial, in which R-CVP resulted the arm with higher rate of events.

Concerning the impact of treatment on the BCL2/IGH molecular tumor burden assessed in 66 of previously $\mathrm{PCR}^{+}$cases, the mean observed reduction was about two logarithms; a lower molecular tumor burden reduction was measured in patients receiving R-CVP versus the remaining ones (decrease $>3 \log =21.1 \%$ for R-CVP vs. $36.8 \%$ for R-FM and $42.1 \%$ for R-CHOP, $P=0.07$ ).

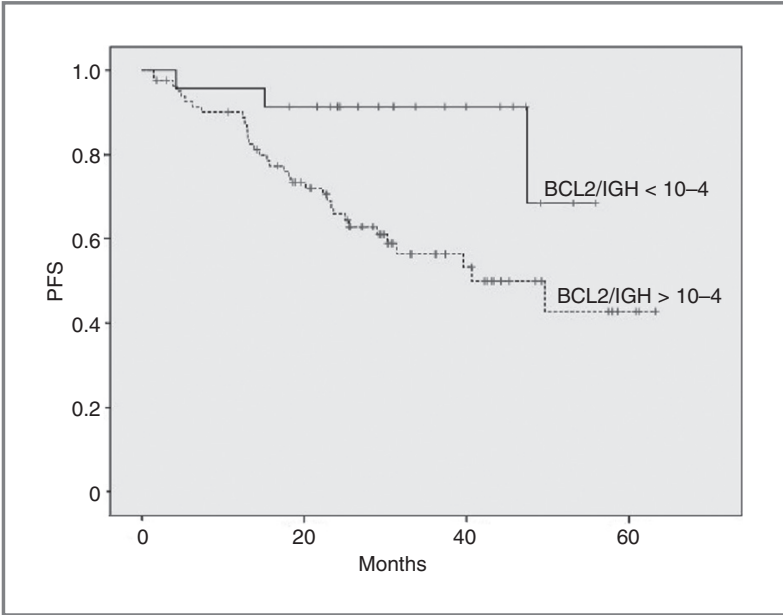

Figure 1. PFS from the randomization is significantly longer in patients with $B C L 2 / / G H$ levels $<1 \times 10^{-4}$ before treatment (continuous dotted line; $P=0.015)$.

The conversion to MRD negativity just after treatment correlated with a lower probability of relapse and longer PFS, but it did not reach a statistical significance (relapse rate, $33 \%$ vs. $41 \% ; P=0.363 ; 3$-year PFS $64.3 \%$ vs. $53.1 ; P=$ $0.08)$.

MRD negativity during follow-up has significant impact on PFS and retains its prognostic significance also in patients achieving partial response

At the molecular assessment performed after 12 months from the end of treatment, 63 cases were MRD ${ }^{-}$, whereas 24 were still $\mathrm{MRD}^{+}$; after 24 months, 46 cases became $\mathrm{MRD}^{-}$, whereas 19 retained their MRD positivity. The allocation of patients in the three arms of therapy was not different between $\mathrm{MRD}^{+}$and $\mathrm{MRD}^{-}$cases.

PFS was significantly conditioned by the PCR status at 12 and 24 months, with 3 -year PFS of $66 \%$ for $\mathrm{PCR}^{-}$cases

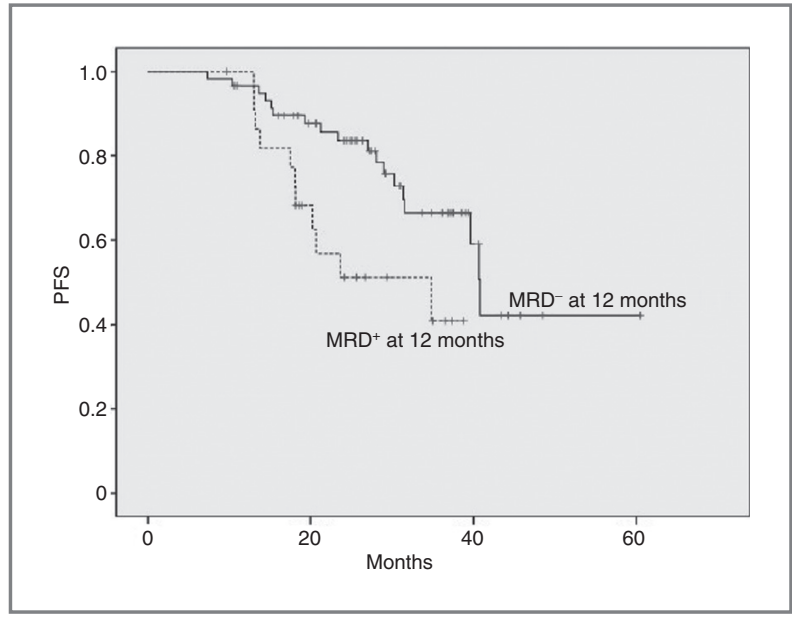

Figure 2. PFS from the randomization is significantly longer in patients without BCL2/IGH detectable after 12 months of follow-up (continuous dotted line; $P=0.015$ ) 


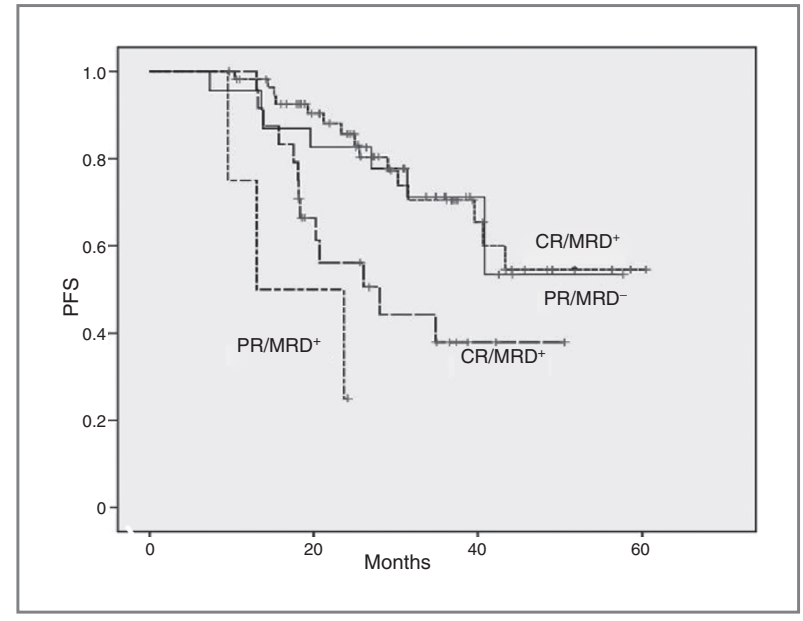

Figure 3. PFS from the randomization is significantly longer in patients without $B C L 2 / / G H$ rearrangement during follow-up, independently of the quality of response; $P=0.001$.

versus $41 \%$ for those $\mathrm{PCR}^{+}$at 12 months ( $P=0.015$; Fig. 2$)$, and $84 \%$ versus $50 \%$ at 24 months $(P=0.014)$.

The MRD negativity at 12 and 24 months from the end of treatment resulted in an improved PFS both in CR and in PR patients (3-year PFS $=72 \%$ for cases CR/PCR ${ }^{-}$vs. $32 \%$ for those CR/PCR ${ }^{+}$vs. $62 \%$ for those PR/PCR ${ }^{-}$and $25 \%$ for patients in $\mathrm{PR} / \mathrm{PCR}^{+} ; \mathrm{P}=0.001$; Fig. 3 ).

When the analysis was restricted to the cohort of patients who achieved CR but then relapsed, the MRD negativity at 12 and 24 months still retained its favorable prognostic significance [3-year $\mathrm{PFS}=88 \%$ for $\mathrm{MRD}^{-}$cases vs. $52 \%$ for those still $\mathrm{MRD}^{+}$at 12 months $(P=0.046)$, and $91 \%$ vs. $52 \%$ at 24 months $(P=0.034)]$.

When PCR negativity at 12 and 24 months was considered in multivariate analysis together with FLIPI, BM involvement, quality of response (CR vs. PR) or stable disease, and arm of therapy (R-CVP vs. R-CHOP or R-FM), only the BM involvement at 12 months retained its poor prognostic role in addition to the MRD persistence $\left(\mathrm{BM}^{+}\right.$: $\mathrm{HR}, 3.23 ; 12$ months $\mathrm{MRD}^{-}: \mathrm{HR}, 0.38 ; P=0.010$ and 0.016 , respectively; see Supplementary Table S2). At 24 months, only the persistence of the BCL2/IGH rearrangement conditioned the long-term outcome (24 months $\mathrm{MRD}^{-}$: HR, 0.26 ; 95\% CI, 0.07-0.92; $P=0.036$; see Supplementary Table S3).

Moreover, when the prognostic role of the MRD at 12 and 24 months was analyzed in respect of the arm of therapy, the Mantel-Heanzel test confirmed that the MRD negativity was a good prognostic factor, independently from the type of treatment (HR, 0.44; test for unequal HR: $P=0.604$ ).

Finally, from the 26 cases still $\mathrm{MRD}^{+}$after treatment, 13 (50\%) achieved the MRD negativity during follow-up; on the other hand, 21 of the 45 cases $\mathrm{MRD}^{-}$after induction (46\%) presented at least one positive PCR during follow-up. There was a good trend to a higher percentage of patients relapse free at 36 months in the cohort of the $\mathrm{MRD}^{+/-}$cases
(76\%) versus cases $\mathrm{MRD}^{-/+}(46 \%)$ versus patients always $\mathrm{MRD}^{+}(21 \%) ; P=0.08$.

When 10 cases with both molecular and clinical relapse were evaluated, in 9 of them the molecular relapse preceded the clinical one for a median of 5 months (range, 2-8).

\section{Discussion}

The results of this large, prospective, and randomized trial support the usefulness of the MRD evaluation in patients with follicular lymphoma treated with conventional chemoimmunotherapy. Four main findings arose from this study: (i) the presence of the BCL2/IGH rearrangement in the $\mathrm{BM}$ at diagnosis has got a predictive value on PFS; (ii) a low molecular tumor burden at diagnosis positively impacts on the quality of response and PFS; (iii) the MRD negativity after 12 and 24 months off treatment correlates with a better outcome; (iv) R-CVP is the regimen offering a lower molecular disease clearance in comparison with R-CHOP and R-FM.

It is relevant that our study compares favorably with other reports because of its larger number of patients: indeed, the majority of reports included not more than 100 cases (12-14). Our study analyzed 415 cases before treatment (105 by qPCR), and 207 during the further 24 months of follow-up, for overall 1,100 PCR reactions.

Another relevant item is represented by the study design: although other studies put together results coming from both peripheral blood and $\mathrm{BM}$, often regardless of the presence of the molecular marker at diagnosis (14), in our study only patients $\mathrm{PCR}^{+}$at diagnosis (always assessed on BM) were considered for the statistical analysis. This could be relevant, because differences higher than 1 log have been reported in favor of BM samples (23). Moreover, qPCR assays were conducted according to the European EUROMRD guidelines (24).

BCL2/IGH rearrangement was detected at diagnosis in $53 \%$ of our patients; this percentage could be considered too low for a marker with prognostic implications; nevertheless, it is worth to consider that in the next future the availability of primers and probes for detecting the rare $B C L 2 / I G H$ breakpoints will increase the number of follicular lymphoma cases with a molecular marker at diagnosis. Moreover, the percentage of cases with a molecular marker is superimposable to that previously reported by other authors $(48 \%-54 \%$; refs. $(14,15,22)$.

Obviously, we have to consider that the BCL2/IGH rearrangement was detected in $2 \%$ to $23 \%$ of healthy donors $(25,26)$. In our study, all data come from the BM analysis only and the specificity of rearrangement was confirmed also by DNA sequencing.

About the clinical impact of the qualitative PCR at diagnosis, $61.9 \%$ of $\mathrm{PCR}^{+}$did not reach $\mathrm{CR}$ versus $38.1 \%$ of the $\mathrm{PCR}^{-}$cases.

As second item, we demonstrated that qPCR, already at diagnosis, is able to predict the outcome of patients with follicular lymphoma. Some authors reported that levels of 
BCL2/IGH $<1 \times 10^{-3}$ did not improve quality of response (14), whereas others showed that cases with low molecular tumor burden at diagnosis achieved CR more frequently than those with high molecular tumor burden (15). In our study, we demonstrated that cases displaying values $<1 \times$ $10^{-4}$ showed a clear advantage in terms of PFS (3-year PFS $80 \%$ vs. $59 \%$ for cases with higher molecular tumor burden; $P=0.015)$ and ORR $(76.6 \%$ vs. $38.9 \% ; P=0.006)$.

Moreover, we reported that the disappearance of the molecular marker after therapy positively conditioned the outcome, with a statistical significance in the long-term follow-up. The lower significance of the molecular marker disappearance at the end of treatment could be justified by the short interval between the last cycle of rituximab and the MRD assessment; indeed, the long-lasting activity of the anti-CD20 antibody could underestimate the MRD ${ }^{+}$cases.

Interestingly, our data sustain the role of the MRD negativity not only in patients reaching CR, but also in those with partial response: 3-year PFS was $62 \%$ for patients in PR and $\mathrm{MRD}^{-}$versus $32 \%$ for patients in CR but still $\mathrm{MRD}^{+}$after 12 months of follow-up. This is interesting, because it seems to be a real proof of the importance of the MRD in follicular lymphoma. Moreover, our data are comparable with those reported by the Nordic Group in mantle cell lymphoma and by our group in follicular lymphoma, in which MRD was highly predictive for prolonged response duration also in cases achieving PR $(27,28)$.

The fourth finding of this study is that patients treated with R-CVP had an inferior clearance of the molecular disease; these data are in perfect accordance with those from the clinical trial in which PFS and time-to-treatment-failure were shorter for the R-CVP arm (17).

In conclusion, our study sustains the importance of the $B C L 2 / I G H$ detection at diagnosis and the utility of the MRD monitoring during the follow-up of patients with follicular lymphoma.

All of us agree that FLIPI and FLIPI2 are very good prognostic factors in follicular lymphoma $(29,30)$; nevertheless, a molecular assessment during the work-up of these kind of patients could be considered such as a sort of "dynamic" risk score that could lead to treat by rituximab patients losing MRD negativity or to avoid maintenance in patients at very low risk of relapse.

PET scan is another tool that proved to be highly predictive for outcome in follicular lymphoma. In our study, we had a small subset of patients in which both tools were used. This panel of patients is too small to be conclusive, but we noticed that PCR was able to discriminate two prognostic subgroups among PET $^{-}$cases, with MRD negativity associated with longer PFS (Luminari S; unpublished data).

\section{References}

1. Ladetto M, Corradini $P$, Vallet S, Benedetti F, Vitolo U, Martelli M, et al. High rate of clinical and molecular remissions in follicular lymphoma patients receiving high-dose sequential chemotherapy and autografting at diagnosis: a multicenter, prospective study by
Moreover, we could speculate that the predictive value of MRD could be jeopardized by the use of rituximab maintenance that is now the standard of care in follicular lymphoma. However, a recent study from our group in old patients receiving R-FND followed by a brief consolidation with rituximab and a random between rituximab maintenance or observation showed power that MRD still retained an excellent prognostic discrimination among patients receiving rituximab maintenance (15). Moreover, our data suggest that a preemptive strategy similar to that used by the Nordic group in mantle cell lymphoma might appear of interest for future studies in follicular lymphoma, as also shown by the retrospective experience from our group (28).

In this line, the FIL recently started a new large randomized phase III trial based on the MRD and PET status assessment after R-CHOP induction (FOLL12-EUDRACT NUMBER: 2012-003170-60, Clinical trial.gov NCT00774826).

\section{Disclosure of Potential Conflicts of Interest}

U. Vitolo reports receiving speakers bureau honoraria from Celgene and Roche. No potential conflicts of interest were disclosed by the other authors.

\section{Authors' Contributions}

Conception and design: S. Galimberti, S. Luminari, M. Ladetto, G.A Palumbo, A. Pulsoni, M. Federico

Development of methodology: S. Luminari, E. Ciabatti, S. Grassi F. Guerrini, M. Ladetto

Acquisition of data (provided animals, acquired and managed patients, provided facilities, etc.): S. Galimberti, S. Luminari, E. Ciabatti, F. Guerrini, C. Mannu, I.D. Giudice, L. Arcaini, A. Tucci, G.A. Palumbo, L. Rigacci, A. Pulsoni, U. Vitolo, C. Boccomini, D. Vallisa, G. Bertoldero, P. Musto

Analysis and interpretation of data (e.g., statistical analysis, biostatistics, computational analysis): S. Galimberti, S. Luminari, A. Dondi, L. Marcheselli, P.P. Piccaluga, A. Gazzola, B. Mantoan, I.D. Starza, M. Cavalli, U. Vitolo, G. Gaidano, M. Federico

Writing, review, and/or revision of the manuscript: $S$. Galimberti, S. Luminari, P.P. Piccaluga, I.D. Giudice, G.A. Palumbo, A. Pulsoni, U. Vitolo, C. Boccomini, G. Gaidano, P. Musto, M. Petrini, M. Federico Administrative, technical, or material support (i.e., reporting or organizing data, constructing databases): $\mathrm{S}$. Galimberti, F. Guerrini, A. Dondi, L. Monitillo, I.D. Starza, M. Cavalli, L. Rigacci

Study supervision: S. Galimberti, S. Luminari, I.D. Giudice

Performed PCR and interpretation of data: I.D. Starza, M. Cavalli

\section{Acknowledgments}

The authors thank Dr. Rossana Testi for her precious samples management and for her help.

\section{Grant Support}

This study was partially supported by grants from the Associazione Angela Serra per la Ricerca sul Cancro, Modena, from AIL Pisa, and by an unrestricted grant from "Ministero della Salute, Dipartimento dell'Innovazione-Direzione Generale Ricerca Scientifica e Tecnologica" (Progetto di Ricerca Finalizzata 2008, IRCCS CROB Rionero CUP J65J08000090000)

The costs of publication of this article were defrayed in part by the payment of page charges. This article must therefore be hereby marked advertisement in accordance with 18 U.S.C. Section 1734 solely to indicate this fact.

Received February 17, 2014; revised August 28, 2014; accepted September 21, 2014; published OnlineFirst October 14, 2014 the Gruppo Italiano Trapianto Midollo Osseo (GITMO). Blood 2002; 100:1559-65.

2. Galimberti S, Guerrini F, Morabito F, Palumbo GA, Di Raimondo F, Papineschi F, et al. Quantitative molecular evaluation in autotransplant 
programs for follicular lymphoma: efficacy of in vivo purging by rituximab. Bone Marrow Transplant 2003;32:57-63.

3. Apostolidis J, Gupta RK, Grenzelias D, Johnson PW, Pappa VI, Summers KE, et al. High-dose therapy with autologous bone marrow support as consolidation of remission in follicular lymphoma: longterm clinical and molecular follow-up. J Clin Oncol 2000;18:527-36.

4. Procházka V, Papajík T. Molecular remission in follicular lymphoma: is the era of residual disease monitoring over? J Clin Oncol 2011; 29:e318.

5. Ferrero $\mathrm{S}$, Drandi $\mathrm{D}$, Mantoan $\mathrm{B}$, Ghione $\mathrm{P}$, Omedè $\mathrm{P}$, Ladetto $\mathrm{M}$. Minimal residual disease detection in lymphoma and multiple myeIoma: impact on therapeutic paradigms. Hematol Oncol 2011;29: 167-76.

6. Pezzella F, Mason DY. The bcl-2 gene and 14;18 translocation in lymphoproliferative disorders. Nouv Rev Fr Hematol 1990;32:397-9.

7. von Neuhoff N, Dreger $P$, Suttorp M, Marget M, Kell S, Schmitz N. Comparison of different strategies of molecular genetic monitoring following autologous stem cell transplantation in patients with follicular lymphoma. Bone Marrow Transplant 1998;22:161-6.

8. Weinberg OK, Ai WZ, Mariappan MR, Shum C, Levy R, Arber DA. "Minor" BCL2 breakpoints in follicular lymphoma: frequency and correlation with grade and disease presentation in 236 cases. $\mathrm{J} \mathrm{Mol}$ Diagn 2007;9:530-7.

9. Kokovic I, Novakovic BJ, Grazio SF, Novakovic S. Sensitivity and reproducibility of conventional qualitative and quantitative PCR assays for detection of the $t(14 ; 18)(q 32 ; q 21)$ chromosomal translocation in biopsy material from patients with follicular lymphoma. Int J Mol Med 2009;23:9-15

10. van Dongen JJ, Langerak AW, Brüggemann M, Evans PA, Hummel M, Lavender FL, et al. Design and standardization of PCR primers and protocolos for detection of clonal immunoglobulin and T-cell receptor gene recombinations in suspect lymphoproliferationc: report of the BIOMED-2 Concerted Action BMH4-CT98-3936. Leukemia 2003;17: 2257-317.

11. Galimberti S, Brizzi F, Mameli M, Petrini M. An advantageous method to evaluate $\mathrm{lg} \mathrm{H}$ rearrangement and its role in minimal residual disease detection. Leuk Res 1999;23:921-9.

12. Rambaldi A, Lazzari M, Manzoni C, Carlotti E, Arcaini L, Baccarani M, et al. Monitoring of minimal residual disease after $\mathrm{CHOP}$ and rituximab in previously untreated patients with follicular lymphoma. Blood 2002;99:856-62.

13. Goff L, Summers K, lqbal $S$, Kuhlmann J, Kunz M, Louton $T$, et al. Quantitative PCR analysis for $\mathrm{Bcl}-2 / \mathrm{lgH}$ in a phase III study of Yttrium90 Ibritumomab Tiuxetan as consolidation of first remission in patients with follicular lymphoma. J Clin Oncol 2009;27:6094-100.

14. van Oers $M H$, Tönnissen $E$, Van Glabbeke $M$, Giurgea L, Jansen $J H$, Klasa $\mathrm{R}$, et al. BCL-2/lgH polymerase chain reaction status at the end of induction treatment is not predictive for progression-free survival in relapsed/resistant follicular lymphoma: results of a prospective randomized EORTC 20981 phase III intergroup study. J Clin Oncol 2010;28:2246-52.

15. Ladetto M, Lobetti-Bodoni C, Mantoan B, Ceccarelli M, Boccomini C, Genuardi $\mathrm{E}$, et al. Persistence of minimal residual disease in the bone marrow predicts outcome in follicular lymphomas treated with a rituximab-intensive program. Blood 2013;122:3759-66.

16. Rambaldi A, Carlotti E, Oldani E, Della Starza I, Baccarani M, Cortelazzo S, et al. Quantitative PCR of bone marrow BCL2/IGH ${ }^{+}$cells at diagnosis predicts treatment response and long-term outcome in follicular non-Hodgkin lymphoma. Blood 2005;105:3428-33.

17. Federico M, Luminari S, Dondi A, Tucci A, Vitolo U, Rigacci L, et al. RCVP versus $\mathrm{R}-\mathrm{CHOP}$ versus $\mathrm{R}-\mathrm{FM}$ for the initial treatment of patients with advanced-stage follicular lymphoma: results of the FOLL05 trial conducted by the Fondazione Italiana Linfomi. J Clin Oncol 2013;31: 1506-13.

18. Barosi G, Carella A, Lazzarino M, Marchetti M, Martelli M, Rambaldi A et al. Management of nodal diffuse large B-cell lymphomas: practice guidelines from the Italian Society of Hematology, the Italian Society of Experimental Hematology and the Italian Group for Bone Marrow Transplantation. Haematologica 2006;91:96-103.

19. Cheson BD, Pfistner B, Juweid ME, Gascoyne RD, Specht L, Horning $\mathrm{SJ}$, et al. Revised response criteria for malignant lymphoma. J Clin Oncol 2007;25:579-86.

20. Gribben JG, Neuberg D, Freedman AS, Gimmi CD, Pesek KW, Barber $M$, et al. Detection by polymerase chain reaction of residual cells with the bcl-2 translocation is associated with increased risk of relapse after autologous bone marrow transplantation for B-cell lymphoma. Blood 1993;81:3449-57.

21. Buchonnet $G$, Lenain $P$, Rumiry $P$, Lepretre $S$, Stamatoullas $A$, Parmentier $\mathrm{F}$, et al. Characterisation of BCL2-JH rearrangements in follicular lymphoma: PCR detection of $3^{\prime}$ BCL2 breakpoints and evidence of a new cluster. Leukemia 2000;14:1563-9.

22. Ladetto M, Sametti S, Donovan JW, Ferrero D, Astolfi M, Mitterer M, et al. A validated real-time quantitative PCR approach shows a correlation between molecular tumor burden and successful ex vivo purging in follicular lymphoma patients. Exp Hematol 2001;29: 183-193.

23. Léonard BM, Hétu F, Busque L, Gyger M, Bélanger R, Perreault C, Roy DC. Lymphoma cell burden in progenitor cell grafts measured by competitive polymerase chain reaction: less than one log difference between bone marrow and peripheral blood sources. Blood 1998;91:331-9.

24. van der Velden VH, Cazzaniga G, Schrauder A, Hancock J, Bader P, Panzer-Grumayer ER, et al. Analysis of minimal residual disease by lg/ TCR gene rearrangements: guidelines for interpretation of real-time quantitative PCR data. Leukemia 2007;21:604-11.

25. Summers KE, Goff LK, Wilson AG, Gupta RK, Lister TA, Fitzgibbon J. Frequency of the $\mathrm{Bcl}-2 / \mathrm{lgH}$ rearrangement in normal individuals: implications for the monitoring of disease in patients with follicular lymphoma. J Clin Oncol 2001;19:420-4.

26. Ladetto M, Drandi D, Compagno M, Astolfi M, Volpato F, Voena C, et al $\mathrm{PCR}$-detectable nonneoplastic $\mathrm{Bcl}-2 / \mathrm{lgH}$ rearrangements are common in normal subjects and cancer patients at diagnosis but rare in subjects treated with chemotherapy. J Clin Oncol 2003;21:1398-403.

27. Pott C, Hoster E, Delfau-Larue MH, Beldjord K, Böttcher S, Asnafi V, et al. Molecular remission is an independent predictor of clinical outcome in patients with mantle cell lymphoma after combined immunochemotherapy: a European MCL intergroup study. Blood 2010;115: 3215-23.

28. Ladetto M, Lobetti-Bodoni C, Mantoan B, Evangelista A, Boccomini C, Genuardi $E$, et al. PCR-based minimal residual disease (MRD) detection is a strong independent outcome predictor also in rituximabintensive non-ASCT-based programs: results from the ML17638 multicenter randomised phase III trial for elderly follicular lymphoma (FL) patients of the fondazione italiana linfomi (FIL) blood (ASH Annual Meeting Abstracts), 2012;120:787.

29. Giné E, Montoto S, Bosch F, Arenillas L, Mercadal S, Villamor N , et al. The follicular lymphoma international prognostic index (FLIPI) and the histological subtype are the most important factors to predict histological transformation in follicular lymphoma. Ann Oncol 2006;17: 1539-45.

30. Arcaini L, Merli M, Passamonti F, Rizzi S, Ferretti V, Rattotti S, et al Validation of follicular lymphoma international prognostic index 2 (FLIPI2) score in an independent series of follicular lymphoma patients. Br J Haematol 2010;149:455-57. 